\title{
The post-pandemic future
}

\author{
By Sharif Islam, Semi-retired Associate, London, UK
}

W ell, I can't help but be a little disappointed. The neonoir, high-tech future I was promised in my youth simply hasn't materialised. A world of neon lights, flying cars, hypersonic travel and bases on the moon are still confined to the realms of movie fantasy. A world run by automation and artificial intelligence, laser scans and holograms, is only a partial reality. Instead, 30 years later, we're mostly still driving the internal combustion engine, we haven't returned to the moon in nearly 50 years (at the time of writing), and computers are simply a more efficient delivery system for pornography, scorn and conspiracy.

Much of dentistry is as it was too. The decades-old dental instruments behind the glass in the Science Museum could easily have been snatched from the pouches in your cabinets. Admittedly, they were designed for a human anatomy that hasn't evolved much since, but still, no fingerprint activated handpieces or laser guided forceps yet.
And what of the post-pandemic future of dentistry? Surely, someone must be considering it. In the event of an apocalypse some of my colleagues understandably vote for a bunker full of beefcake but, alas, you need a few thinkers in there too. Someone to invent the telephone, perhaps. And after COVID-19 has taken its leave of the spotlight and started the long journey towards being a footnote of history, how will dental practice have changed?

Will using a drill remain an exercise in PPE-induced autoasphyxiation, fraught with the stress over generating a lingering aerosol? Will our diligent hygienists still be expected to suffer repetitive strain injury by scaling only by hand? Will our patients still have to form a socially-distanced queue out into oncoming traffic? And will an innocent cough or sneeze still be followed by an awkward silence and anxious glances?

Undoubtedly, our council of chiefs will determine if and when we can return to a more normal form of practice, whatever normal is eventually deemed to be. But I doubt it will so easily or 
« promptly be a casual return to the pre-COVID era, a wonderful time in human history when toilet paper had no good reason to make headline news. Since then, the pandemic has incentivised us to implement practices that are actually worth keeping. And, evidently it seems, to stockpile reams of toilet roll.

It may be a while before everyone is entirely comfortable to sit in the waiting room without a mask. But I suspect the hand sanitiser units at the entrance will remain, as will the screens at reception to prevent patients' spittle flying across the void when balking at the

\section{'Our conceit at taking life for granted has been bevelled and chamfered into a smoother preparation, soon to be covered by a crown of greater humility.'}

cost of their treatment. We will surely cast an even more fastidious eye over our disinfection and cross infection protocols, ensuring their robustness not only for patient and staff safety, but also for their peace of mind too.

There will also be other more abstract strands unfurling from this era. Dental professionals dedicate themselves to the service of their communities, often eschewing the stress and sacrifice inevitable to that undertaking. Perhaps some will now have a more philosophical view of their lives, attaching new significance to their mortality and desiring more balance to their daily grind. They will have rediscovered their children through home-schooling, noticing that they too may be riven with self-doubt and identity crises, amplified by the brutality of social media. They may also, however, rediscover their spouses and partners, and then hastily retreat back to the shelter of work.

For all these reasons and more, the future of dentistry has greater potential than ever before in its epic saga, possibly since G. V. Black first postulated cavity design on the back of a napkin. Our procedures and our attitudes to work have evolved for the better, offering such effective protection and cleanliness that we wonder why it took an entire pandemic to make that happen.

Our understanding and empathy of mental health has by unfortunate necessity improved, as has our ability to educate and reassure our patients. Our conceit at taking life for granted has been bevelled and chamfered into a smoother preparation, soon to be covered by a crown of greater humility.

Most importantly, perhaps, we now know with the utmost confidence that we can weather any future pandemic. To quote the Six Million Dollar Man, we have the technology. Our practices can remain open as a vital service to help our patients through even the most trying of times. Learning that lesson, as frustrating as it may have been, was surely worthwhile.

So, let's be bold enough to play the long game and see this through. Even just a little of that perseverance we ask of our patients will help us endure this long pandemic procedure. Wince, by all means, but let's be sure to open our eyes once the pain wears off. Our future will be there waiting for us. 